\title{
Spatio-Temporal Modelling of Frequent Human Mobility Pattern to Analyse the Dynamics of Epidemic Disease
}

\author{
M. Parimala* \\ Daphne Lopez \\ ${ }^{I}$ VIT University, India \\ * Corresponding author’s Email: parimala.m@ vit.ac.in
}

\begin{abstract}
Spatial data mining is a rapidly growing field for analysing the data related to space and time. Nowadays most of the applications are based on these factors, so numerous data mining algorithms are developed for spatial characterization and to analyse the spatial trends. The spatial trend analysis determines the change in pattern of some non-spatial attributes on neighbourhood objects. In this paper, we identify spatio-temporal mobility pattern on the dynamics of Epidemic disease (H1N1) that plays a significant role in analysing the outbreak of an infectious disease. Modelling the transmission among the human population with respect to time and space leads to improved understanding of transmission mechanisms. A compartmental model is designed to characterize the disease dynamics of a random variable extracted from binomial and multinomial distribution. ArcGIS tool is used to visualize the mobility distribution of the infected host spatially and yields an output of frequent mobility locations with respect to different time slices. The results thus obtained would help the district administrative authorities to take strategic decisions and prevent the spread of the disease.
\end{abstract}

Keywords: Multinomial distribution; frequent; mobility pattern; GIS; dynamics; epidemic model; spatio-temporal

\section{Introduction}

Spatio-temporal field in Data Mining has gained high popularity in geographic data sciences because of the pervasiveness of a wide range of location based or ecological gadgets that record position, time or/and natural properties of an item or set of objects in real time. So, different approach is required to deal with knowledge discovery and the challenges in information analysis for different kinds of spatio-temporal data. Adapting more about individuals mobility is an essential task for administrative leaders and urban organizers. Mobility information describes the properties of objects in various locations over time and in addition movements of individuals between the locations. The investigation of mobility data is a difficult task to understand the spatio-temporal changes due to its different spatial location. For each object in each time period, there is more number of measurements associated with them, which is not feasible to analyze every measurement in a complex time series. Thus the series of time are analyzed in terms of time slices that represent unique temporal features within which discovery of pattern analysis can be focused. Tracking the spatial change over the time intervals is a challenging and difficult task. The main motivation of this paper is to identify the change and dynamics in spatial-temporal pattern analysis over the mobility of objects in Health Analytics.

2009, H1N1 (swine flu) is a new influenza virus similar to regular seasonal influenza virus that spreads from person-to-person through contact of an infected individual or transportation. Transportation via air traffic has largely contributed to this rapid spread across the globe. The rapidity of the spread depends on the nature of the growing population and the mobility of the infected agent [1]. Though the practice of using concrete data and evidence to support medical decisions has existed for centuries 
[2], the first outbreak of swine flu was discovered only four months after the first outbreak in India. The country's first swine flu death, that of a 14year-old school girl on August 3, 2009 followed by many more patients succumbing to the disease in the span of just over a week set off a panic attack that was spreading faster than the outbreak itself which is now re-emerging in the state.

A total of 3,315 lives have been lost and 27,236 had been infected due to H1N1 in India. In 2010 , the deaths went up to 1,763 while the number of cases remained around 20,000. In 2011 and 2012, 603 and 5,044 persons, respectively, were diagnosed positive for the disease and total 480 deaths were reported. Since January this year, there have been more than 494 cases of Swine Flu and 95 deaths all over the country. Rajasthan tops the list with 54 deaths followed by Gujarat (six), Maharashtra and Delhi (four each). The World Health Organization (WHO) has reported that in the post-pandemic period, India had experienced outbreaks during the period August to October, 2010; May to July 2011; March and October 2012 and also in JanuaryFebruary 2016 (Fact sheet of H1N1,WHO [3]).

In a globalized world, infectious diseases such as SARS, swine flu or seasonal influenza can be spread over the entire planet by travellers. To enable a more effective response to this hazard, researchers are trying to predict the transmission paths and speed of such epidemics. Mathematical models were developed to identify the individual mobility patterns. Human beings are the most complex unknown system to predict epidemics and their transmission. The spread of disease entirely depends on how the infected individual travels. Generally, in traditional models the human mobility patterns [4] were idealised because it was difficult to refine them mathematically. Earlier epidemic models bypassed this problem by applying the simplified assumption that an infection spreads according to the principles of diffusion. Literature studies have proved that it is difficult to reflect all aspects of human mobility in the whole huge population. It simplifies the problem by assuming that each person visit every possible location or it ignores the members of a population, as individuals are considered to reach unique source and destination. This results in an individual mobility network which consists of the limited number of destinations that the person will travel to. In our proposed model, the mobility pattern on locations is identified rather than mobility pattern on individual in the population. Any type of epidemic disease can be classified into various stages namely susceptible (who are not infected), Infected (who are infected with the disease), Recovered and Death compartments. The dynamics of the disease can be determined by number of people who transit from one compartment to other compartment. The transition of human alone is not sufficient to evaluate the dynamics of disease spread. Additionally, it requires analysis of mobility pattern in various locations and time is required to take a good decision for the administrators. follows,

The main contributions of this paper are as

(i) A compartmental model (SLITR) is designed to capture the various disease transition state in the epidemic disease.

(ii) The rate of change of individual in various compartments during the transition of disease is defined using differential equations

(iii) The dynamics of mobility on the epidemic model with respect spatial (location) and temporal (time) aspect is determined based on distributive approach using multinomial distribution.

(iv) The distributive approach is implemented using Matlab and simulated using Quantum GIS software.

(v) The proposed compartmental model (SLITR) is compared with other epidemic model (SIR, SEIR) and proves that the result of the proposed model is closer to the real dataset.

(vi) The significant contribution from this spatiotemporal analysis can be used by the district administrative officers to control the spread or to make decision on prevention strategies like vaccination

The paper is organised as follows, Section 2 discuss about the related work, Modelling the compartmental model is given in Section 3, Implementation and results are discussed in Section 4 and finally Section 5 concludes the work.

\section{Related work}

Recently, mining patterns in spatio-temporal data [5] has been a popular area of research in the field of data mining. The change of pattern [6][7] in data with respect to space and time is considered to be interesting part of spatio-temporal data mining.W.O. Kermack (1927) laid the foundation [8] for modeling the spread of epidemics. The very first mathematical model [9][10] is SIR model where the closed population is subdivided into Susceptibles, Infectives and Recovered individuals. Each infective is infectious for a certain period of time. The infected individual gets contact with the susceptibles who will then become infective. After a certain period, the individual is removed, by immunization or by death. The above model is 
proved by certain assumptions [11] like a total population is kept constant, recovered individuals are not infected again, age and population structure is not considered.

Recent methods of ubiquitous computing have generated large amounts of human behavioural data. The social and human dynamics are key elements for the effective modelling of virus spreading. Traditional epidemiologic models do not capture individual information and hence have a complex nature in the ability of disease spreading process. To overcome this limitation, agent based approaches are used widely to model spread of epidemic disease. In agent based modelling (ABM) individual human behaviour [12][13] and its inherent fuzziness are simulated by representing every person as a software agent. In ABM model each agent is characterized with a variety of variables that are related to spread of disease such as social characteristics, socio-economic status, health status, etc. Even in these approaches the mobility pattern of human or spatio-temporal aspect is not discussed. SIR model is based on Agent Based Modeling (ABM) [14] to model the infectious individual interactions based on space, time, age and population structure. The ability to model the spread of disease is a critical issue for governments and health organizations. As the behaviour of agent depends on the census or survey data they fail to model the spatio temporal dynamics of human behaviour.

The next compartmental model designed specifically for sexually transmitted disease is SIS model where the susceptible individuals become infected and they become susceptible again. A slightly varied model SEIR [15], includes Susceptible individuals who are Exposed to the environment and become Infectious and then enter into the Recovered compartment. The SEIAHR [16] model categorizes the infected individual as symptomatic and asymptomatic infectious individual. The symptomatic individual either go for recovered or treated compartment whereas the asymptomatic individual transit to the recovered compartment. The combination of SEIRD and SEIAHR model is designed as SELMAHRD. In all the models discussed vaccination rate is taken into account but in SVEIR [17] model it refers to the possible states of Susceptible, Vaccination, Exposed, Infected and Recovered where the vaccinated people directly enter into recovered compartment

The compartmental models do not focus on mobility of the individual [18]. In general the deterministic models fail to consider the spatial aspects of spread of an epidemic, individual contact process and the individual behaviour. Global epidemic and mobility computational model [19] uses human mobility patterns at a global scale. The spatial spread of epidemic disease is modelled using the SEIR where the treated and death compartment is not analysed. The proposed compartmental model, analyse the mobility pattern of human population with disease dynamics in all the compartments with respect to time. Different levels of population and human mobility patterns are the most important factors for spread of disease [20][21]. The bidirectional movement of an individual mobility network [22][23] between home and other locations is analyzed for the spread of disease. The mobility of the human can be based on the status of the number of infected individuals in that location [24]. The spatio-temporal pattern for global epidemic is based on the commuting flows within the local and global population [25][26]. Segolene et al., [27] divides the commuting network from residence to work or school and uses road network to find the local spread of disease. Two groups are compared using Jaccard index, where the two districts that are paired in each network is derived. As a result the spatial spread of the disease was driven more by school commuting than by work commuting. Depending on the age category and commuting network different patterns of spread are involved in transmission. But this study fails to analyse the temporal aspects which is one of the key feature in epidemic dynamics. Planning for a pandemic (e.g., $\mathrm{H} 1 \mathrm{~N} 1$, influenza, etc) is a public health priority of any government. Traditional epidemiological approaches are purely based differential equations that divide the population into various subgroups based on the nature and characteristics of the disease. Although they are extremely successful in guiding and making decision in health policies, these models fail to capture the complexity in human mobility

The proposed study models the transmission of disease with epidemic dynamics derived from mobility network with respect to various time slices. The study area includes the densely populated locations like schools, colleges, work place and specifically hospitals. This paper focuses on a new deterministic model for the spread of H1N1 in India one of the densely populated countries in the world. This model considers the mobility of the individual which perhaps, is one of the major strengths to determine the infection rate. Densely populated locations are chosen and the spatial mobility of infected individuals over space and time is considered to analyse the disease dynamics. Given the importance of spatial spread of human infectious diseases, this study demonstrates the crucial role 
played by human mobility in the spread of epidemic disease. A compartmental model with Susceptible, Latent, Infectious and Treatment and Recovered compartments is designed taking into account the immunity of the individual. To characterize the disease dynamics a random variable extracted from binomial and multinomial distribution is used. In order to populate the disease spread model, based on the mobility of the individual road network data of the state is used. Points of origin are selected based on the hospitals recognized for treating H1N1 that is in and around the state of Tamil Nadu, India .A transport rate factor is derived to estimate the mobility of the individual from one location to another based on the number of public transport plying. This transport rate is then integrated with the distribution function to determine the location which has the maximum mobility rate. ArcGIS tool is used to visualize the mobility distribution of the infected host. The following section describes the model and the method of analysis.

\section{Modelling of compartmental model}

Epidemics can be modelled mathematically inorder to study the severity and prevention mechanism for spread of disease. A transmission disease model (SLITR) that is proposed for H1N1 consists of various compartments (Figure 1) such as Susceptible, Latent, Infectious, Treatment and Recovered to compute the number of people in a population at any time. Initial population is considered in the susceptible compartment. The only way the people can leave the susceptible group is to become infected. The people who are infected go to latent and enter into infected compartment when they become infectious. Based on the mobility and symptoms, they are categorized into four compartments. People with symptoms are treated and people without symptoms are not treated. Based on the immunity the treated or non-treated people enter into recovered and death compartment.

\section{Representation of Symbols}

$S_{i} \quad$ Susceptible Individual at location $\mathrm{j}$

$L_{j} \quad$ Latent Individual at location $\mathrm{j}$

$I_{j}^{n t} \quad$ Symptomatic people who do not travel

$I_{j}^{t} \quad$ Symptomatic people who travel

$I_{j}^{a t} \quad$ Asymptomatic people who travel

$I_{j}^{a n t} \quad$ Asymptomatic people who do not travel
$T_{j}^{h} \quad$ People who are treated

$T_{j}^{n h} \quad$ People who recover with low Immunity

$R_{H I} \quad$ People who recover with high Immunity

D People who go to death compartment

\subsection{Geographical Dataset}

The geographical dataset chosen is for Tamil Nadu one of the largest and seventh populous states in India that has the highest number of H1N1 infection according to data (Table 1) provided by Government of Tamil Nadu, India. It has a population of 72,138,958 (2011 census) and covers an area of $130,058 \mathrm{~km}$ sq. It has a total of 32 districts which are divided into 10 city corporations, 125 municipalities, 529 town panchayats and 12,524 village panchayats. In the literature, international air travel has proved to be a good prediction for the worldwide spread of SARS and influenza A/H1N1 2009 , but it was shown that intervention on the global air traffic would be of limited efficacy. At a more local scale, air travel is less relevant and other types of movement must be taken into account. Commuting, i.e. daily movements from residence to work, school, college and hospitals has been widely used to describe spatial mobility in models, using exhaustive datasets or gravity models. Since the spread of disease occurs due to the mobility of the infected individual, we consider road transportation to be the commuting network.

\subsection{Immunity Analysis}

Immune system of human comprises of proteins, tissues and organs that work together to protect our body. Immune cells are made up of white blood cells or leukocytes. There are two types of leukocytes [28] namely B cells and T cells. The B cells produce antibodies and the $\mathrm{T}$ cells determine the immunity level of the person. The immunity level of the individual can be determined using age, vaccination and $\mathrm{T}$ cells factors. The normal value of $\mathrm{T}$ cells range between $500-1500$ cells $/ \mathrm{mm}$. The $\mathrm{T}$ cell count for a H1N1 vaccinated person [29] must be greater than 200 cells $/ \mathrm{mm}$. The age factor is broadly categorized into three types namely category-1 whose age value between 1 and 25 , category-2 (26-55) and category-3 (56-80). Immunity level of an individual either low or high is based on age, vaccination status and $\mathrm{T}$ cell count and is depicted in Figure 2. 


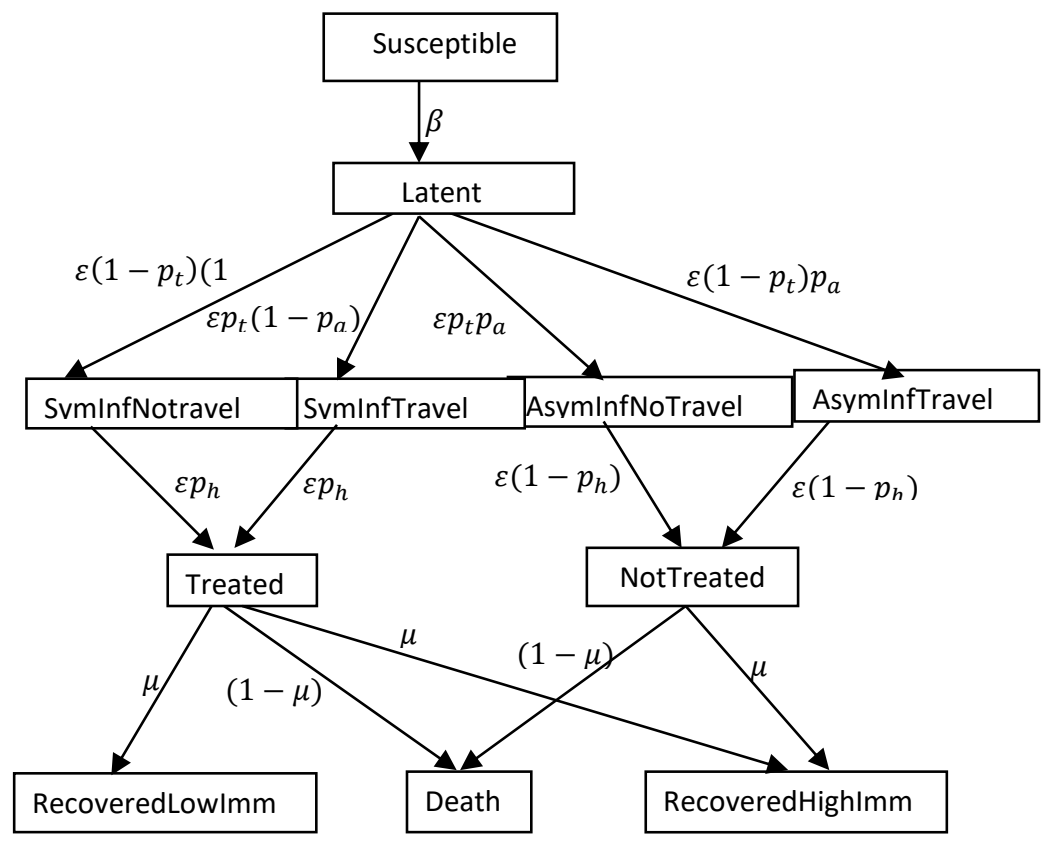

Figure. 1 Compartmental model

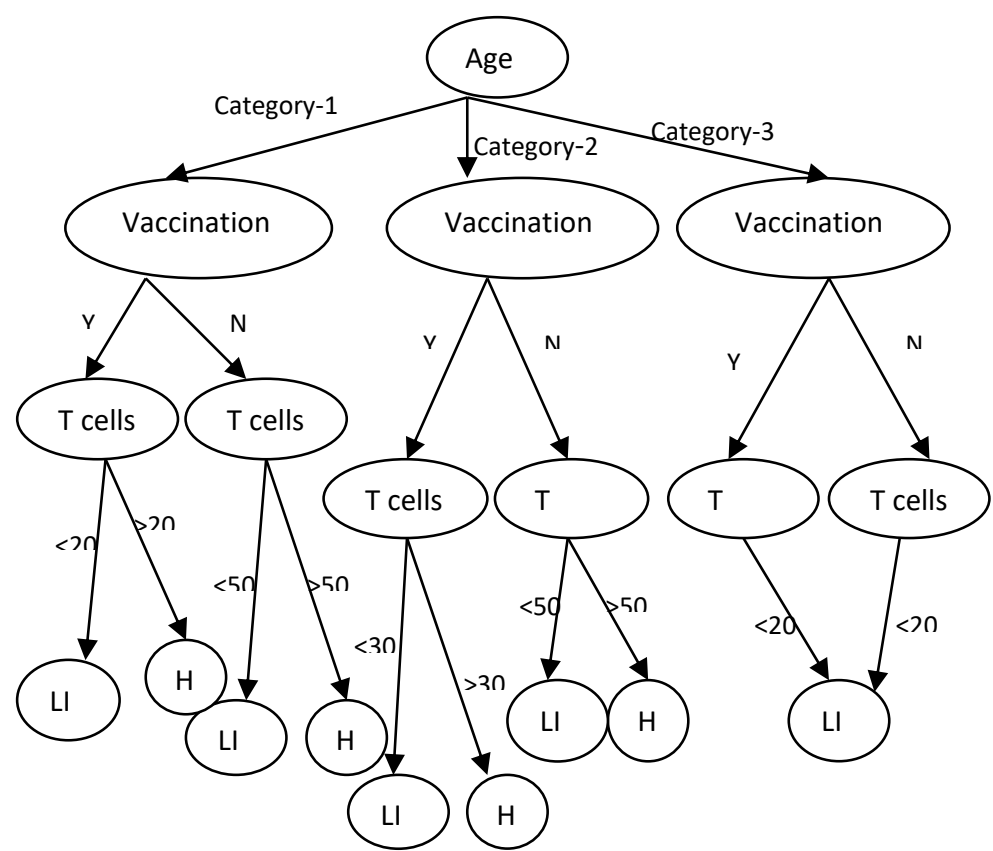

Figure. 2 Decision tree for Immunity 
Table 1. A Report on Influenza A (H1N1) of various districts

\begin{tabular}{|l|l|l|}
\hline Sno & State name & $\begin{array}{l}\text { Number of infected } \\
\text { individual }\end{array}$ \\
\hline 1 & Andra Pradesh & 41 \\
\hline 2 & Kerala & 25 \\
\hline 3 & Maharastra & 2 \\
\hline 4 & Karnataka & 6 \\
\hline 5 & Pune & 2 \\
\hline 6 & Jharkhand & 5 \\
\hline 7 & U.P & 1 \\
\hline 8 & Pondicherry & 1 \\
\hline 9 & Manipur & 1 \\
\hline 10 & Meghalaya & 1 \\
\hline 11 & Jharkhand & 5 \\
\hline 12 & Tamil Nadu & 297 \\
\hline 13 & Others & 45 \\
\hline & Total & 432 \\
\hline
\end{tabular}

\subsection{Transition between the compartments}

The Susceptible population $\left(S_{i}\right)$ enter into latent compartment $\left(L_{j}\right)$ with the probability of $\beta \Delta t$ where $\Delta t$ is the time interval. The symbol $\varepsilon$ denotes a small probability value that selects random action independent of the current action values. The latent individuals either transit to asymptomatic infectious compartment $\left(I_{j}^{a}\right)$ with the probability of $p_{a}$ or they transit to the symptomatic infectious $\left(I_{j}\right)$ compartment with the probability of $\left(1-p_{a}\right)$. People with symptoms are further divided as who travel $\left(I_{j}^{t}\right)$ with the probability of $p_{t}$ and those who do not travel $\left(I_{j}^{n t}\right)$ with the probability of $\left(1-p_{t}\right)$. People without symptoms are also classified as those who travel $\left(I_{j}^{a t}\right)$ and those who do not travel $\left(I_{j}^{\text {ant }}\right)$ with the probability of $p_{t}$ and $\left(1-p_{t}\right)$. The entire symptomatic infectious person enters into treated compartment $\left(T_{j}^{h}\right)$ with the probability of $p_{h}$. The probability at which an asymptomatic infectious person transit into compartment with no treatment $\left(T_{j}^{n h}\right)$ is $\left(1-p_{h}\right)$. The probability of the treated individuals moving into the recovered compartment with high and low immune is $\mu \Delta t$. Individuals who are not treated enters into $R_{H I}$ (recovered with high immune). A person enters the death compartment (D) irrespective of being treated or not treated has a probability of $1-\mu$ in the time step $\Delta \mathrm{t}$. The transition between each compartment is summarized (Table 2) as follows
Table 2. Transition between each compartment

\begin{tabular}{|c|c|}
\hline Transition & Rate \\
\hline$S_{i} \rightarrow L_{j}$ & $\beta$ \\
\hline$L_{i} \rightarrow I_{j}^{t}$ & $\varepsilon p_{t}\left(1-p_{a}\right)$ \\
\hline$L_{i} \rightarrow I_{j}^{n t}$ & $\varepsilon\left(1-p_{t}\right)\left(1-p_{a}\right)$ \\
\hline$L_{i} \rightarrow I_{j}^{a t}$ & $\varepsilon p_{t} p_{a}$ \\
\hline$L_{i} \rightarrow I_{j}^{a n t}$ & $\varepsilon\left(1-p_{t}\right) p_{a}$ \\
\hline$I_{j}^{t}, I_{j}^{n t} \rightarrow T_{j}^{h}$ & $\varepsilon p_{h}$ \\
\hline$I_{j}^{a t}, I_{j}^{a n t} \rightarrow T_{j}^{n h}$ & $\varepsilon\left(1-p_{h}\right)$ \\
\hline$T_{j}^{h} \rightarrow R_{L I}, R_{H I}$ & $\mu$ \\
\hline$T_{j}^{n h} \rightarrow R_{H I}$ & $\mu$ \\
\hline$T_{j}^{h}, T_{j}^{n h} \rightarrow D$ & $(1-\mu)$ \\
\hline
\end{tabular}

SLITR model can be described by the following system of differential equations from Equation (1) to Equation (11). These equations find the number of people in each compartment at time $t$.

$\frac{d S}{d t}=N-\beta S$

$\frac{d L}{d t}=\beta S-\left(\left(1-p_{t}\right)\left(1-p_{a}\right)+p_{t}\left(1-p_{a}\right)+\right.$ $\left.p_{t} p_{a}+\left(1-p_{t}\right) p_{a}\right) L$

$\frac{d I_{n t}}{d t}=\left(\left(1-p_{t}\right)\left(1-p_{a}\right)\right) L-p_{h} I_{n t}$

$\frac{d I_{t}}{d t}=\left(p_{t}\left(1-p_{a}\right)\right) L-p_{h} I_{t}$

$\frac{d I_{\text {ant }}}{d t}=p_{t} p_{a} L-\left(1-p_{h}\right) I_{\text {ant }}$

$\frac{d I_{a t}}{d t}=\left(1-p_{t}\right) p_{a} L-\left(1-p_{h}\right) I_{a t}$

$\frac{d T_{h}}{d t}=p_{h}\left(I_{n t}+I_{t}\right)-\mu T_{h}$

$\frac{d T_{n h}}{d t}=\left(1-p_{h}\right)\left(I_{a n t}+I_{a t}\right)-T_{n h}$

$\frac{d R_{L I}}{d t}=\mu T_{h}$

$\frac{d R_{H I}}{d t}=\mu\left(T_{h}+T_{n h}\right)$

$\frac{d D}{d t}=(1-\mu)\left(T_{h}+T_{n h}\right)$ 


\subsection{Epidemic disease dynamics}

The proposed compartmental model is designed to know the transmission of disease due to mobility of the infected individual. Once the mobility and disease dynamics are defined, the number of individuals in subpopulation $j$ and in each compartment follows a stochastic and discrete dynamic equation that is given as

$C_{j}^{[m]}(t+\Delta t)-\Delta C_{j}^{[m]}(t)=\Delta C_{j}^{[m]}+\varphi_{j}([m])$

where, $\Delta C_{j}^{[m]}$ represents the change due to compartment transition induced by the disease dynamics and transport operator $\varphi_{j}([\mathrm{~m}])$, denotes the variations due to the mobility of individuals over the road commuting network. The transport operator is then integrated with epidemic dynamics with a minimum time scale of one day. The following section discuss about the epidemic transition $\left(\Delta C_{j}^{[m]}\right)$ and integration of mobility in epidemics using transport operator $\varphi_{j}([m]) . \Delta C_{j}^{[m]}$ can be given as a combination of set of operators $D_{j}([m],[n])$ which denotes the number of transitions from compartment $m$ to $n$ in $\Delta t$. This transition is simulated as a random variable extracted from the multinomial and binomial distribution. The $\Delta C_{j}^{[m]}$ is given by the sum of

$\Delta C_{j}^{[m]}=\sum_{n}\left\{-D_{j}\left([m],[n]+D_{j}[n][m]\right)\right\}$

There are four transitions from latent compartment $\left(\boldsymbol{L}_{\boldsymbol{j}}\right)$ such as transitions to the traveling and nontraveling symptomatic infectious and transition to the travelling and non-travelling asymptomatic infectious compartments. The number of transition acting on $\boldsymbol{L}_{\boldsymbol{j}}$ is given by multinomial distribution [30].

$A^{\text {Multi }}\left(L_{j}(t), p_{L_{j}} \rightarrow I_{j}^{t}, p_{L_{j}} \rightarrow I_{j}^{n t}, p_{L_{j}} \rightarrow I_{j}^{a t}, p_{L_{j}} \rightarrow\right.$

$\left.I_{j}^{\text {ant }}\right)$

where the transition probabilities,

$p_{L_{j}} \rightarrow I_{j}^{t}=\varepsilon p_{t}\left(1-p_{a}\right) \Delta t$

$p_{L_{j}} \rightarrow I_{j}^{n t}=\varepsilon\left(1-p_{t}\right)\left(1-p_{a}\right) \Delta t$

$p_{L_{j}} \rightarrow I_{j}^{a t}=\varepsilon p_{t} p_{a} \Delta t$

$p_{L_{j}} \rightarrow I_{j}^{\text {ant }}=\varepsilon\left(1-p_{t}\right) p_{a} \Delta t$

$L_{j}(t)$ represent the number of individual in the latent compartment. Binomial distribution is used to determine the increase in the population of latent compartment

$A^{b i n}\left(S_{j}(t), p_{S_{j}} \rightarrow L_{j}\right.$

where the transition probability is denoted by

$p_{S_{j}} \rightarrow L_{j}=\beta \Delta t$

For a given force of infection $(\beta)$, in subpopulation $j$, each individual in the susceptible compartment $\left(S_{j}\right)$ acquires the infection and enters the latent compartment $\left(L_{j}\right)$ with the probability of $\beta \Delta t$, where $\Delta t$ is the time interval. The number of individuals moving from latent to infectious compartment at time $t$ is generated using probability distribution functions is defined as follows.

$$
\begin{aligned}
& \Delta L_{j}(t)=-\left[D_{j}\left(L, I^{t}\right)+D_{j}\left(L, I^{n t}\right)+D_{j}\left(L, I^{a t}\right)+\right. \\
& \left.D_{j}\left(L, I^{\text {ant }}\right)\right]+D_{j}(S, L)
\end{aligned}
$$

The transition of people from treated $\left(T_{j}^{h}\right)$ to recovered and death compartment is determined using the multinomial distribution

$$
A^{\text {Multi }}\left(T_{j}^{h}(t), p_{T_{j}^{h}} \rightarrow R_{L I}, p_{T_{j}^{h}} \rightarrow R_{H I}, p_{T_{j}^{h}} \rightarrow D\right)
$$

The incoming population for $T_{j}^{h}(t)$ is given by

$$
A^{b i n}\left(p_{I_{j}^{t}} \rightarrow T_{j}^{h}, p_{I_{j}^{n t}} \rightarrow T_{j}^{h}\right)
$$

So the total transition in treated compartment $T_{j}^{h}(t)$ is given by,

$$
\begin{aligned}
& \Delta T_{j}^{h}(t)=-\left[D_{j}\left(T^{h}, R_{L I}\right)+D_{j}\left(T^{h}, R_{H I}\right)+\right. \\
& \left.D_{j}\left(T^{h}, D\right)\right]+\left[D_{j}\left(I_{j}^{n t}, T^{h}\right)+D_{j}\left(I_{j}^{t}, T^{h}\right)\right]
\end{aligned}
$$

Similarly the people moving from non-treated population $\left(T_{j}^{n h}\right)$ to recovered and death compartment is determined by

$$
\begin{aligned}
& \Delta T_{j}^{n h}(t)=-\left[D_{j}\left(T^{n h}, R_{H I}\right)+D_{j}\left(T^{h}, D\right)\right]+ \\
& {\left[D_{j}\left(I_{j}^{a t}, T^{n h}\right)+D_{j}\left(I_{j}^{a n t}, T^{n h}\right)\right]}
\end{aligned}
$$

\subsection{Integration of mobility with the epidemic model}

Mobility of the individual is considered through common road network where $\delta_{j k}$ represents the number of buses available between the bus stops $(j, k)$. Considering the uncertainty of the number of passengers at a particular time a random number $(\theta)$ 
is generated between the interval $(-1,1)$ at time $t$. We assume that on each route $(j, k)$, the stochastic variable for the flux of passengers at time $t$ is given by

$\widetilde{\delta_{j k}}=\Delta \delta_{j k}[\alpha+\theta(1-\alpha)]$

where $\alpha$ is the average occupancy rate (70-90\%). The probability of each individual $C_{j}^{[m]}$ in compartment $m$ traveling from $j$ to $k$ location is $p_{j k}=\frac{\widetilde{\delta_{k}} \Delta t}{N_{j}}$ where $N_{j}$ denotes the number of people in subpopulation $j$. The number of individuals traveling $\left(\xi_{j k}\right)$ from the subpopulation $\mathrm{j}$ on each route $j \rightarrow k$ at time $t$ is defined using multinomial distribution.

$$
P\left(\left\{\xi_{j k}\right\}\right)=\frac{c_{j}^{[m]} !}{\left(c_{j}^{[m]}-\sum_{k} \xi_{j k}\right) ! \Pi_{k} \xi_{j k} !} \prod_{k} p_{j k} \xi_{j k} \times
$$

Where

$C_{j}^{[m]}$ is the number of individuals in compartment $m$ at $j$ location

$C_{j}^{[m]}-\sum_{k} \xi_{j k}$ is the number of individual who do not travel

$\xi_{j k}$ is the number of people who travel from $j$ to $k$

The multinomial distribution $P\left(\left\{\xi_{j k}\right\}\right)$ gives the probability of individual traveling from $j$ to all the other possible routes. Now, the transport operator at location $j$ in compartment $m$ is defined by,

$\varphi_{j}([m])=\sum_{k}\left(\xi_{k j}\left(C_{k}^{[m]}\right)-\xi_{j k}\left(C_{j}^{[m]}\right)\right)$

The value generated from equation (23) is substituted in equation (12) to find the number of people moving from subpopulation $j$ to $k$ for each compartment.

\section{Results and Discussion}

The geographic space considered for the study includes Vellore district and Chennai. The data on the locations is collected from the National Informatics centre (NIC) of Vellore district. Table 3 below shows the source and destination locations $j$ and $\left(k_{1,2 \ldots 10}\right)$ respectively, that are considered for determining the mobility of the individuals in the infected $(m)$ compartment. One of the densely populated location, Christian Medical College (CMC) hospital is an authorized H1N1 influenza treatment centre and the locations in and around the Vellore district is considered as source and destination locations.

Temporal pattern of a patient in a day is sliced in five intervals namely $\mathrm{t} 1(7 \mathrm{am}-9 \mathrm{am}), \mathrm{t} 2(9$ am- $12 \mathrm{pm}), \mathrm{t} 3(12 \mathrm{pm}-3 \mathrm{pm}), \mathrm{t} 4(3 \mathrm{pm}-6 \mathrm{pm})$ and t5(6 pm-9 pm). The mobility of an infected individual through this time interval from source location $j$ to destination locations $k_{i}$ and vice versa is collected for the month of August, 2012. For each time slice, data of symptomatic and asymptomatic infected population who travel is collected and scaled as 0 to 4 using min-max normalization technique. If there is more mobility of infected population, it denotes 4 and 0 if there is no mobility of infected individual.

Table 3. $j$ represents the source location, $k_{1}, k_{2} \ldots k_{10}$ represents destination location

\begin{tabular}{|l|l|}
\hline & \multicolumn{1}{|c|}{ Location name } \\
\hline$j$ & $\begin{array}{l}\text { CMC } \\
\text { Hospital,Vellore }\end{array}$ \\
\hline$k_{1}$ & Tirupattur \\
\hline$k_{2}$ & Vaniyambadi \\
\hline$k_{3}$ & Ambur \\
\hline$k_{4}$ & Gudiyattam \\
\hline$k_{5}$ & Katpadi \\
\hline$k_{6}$ & Wallaja \\
\hline$k_{7}$ & Arcot \\
\hline$k_{8}$ & Arrakonam \\
\hline$k_{9}$ & Chennai \\
\hline$k_{10}$ & Tiruvallur \\
\hline
\end{tabular}




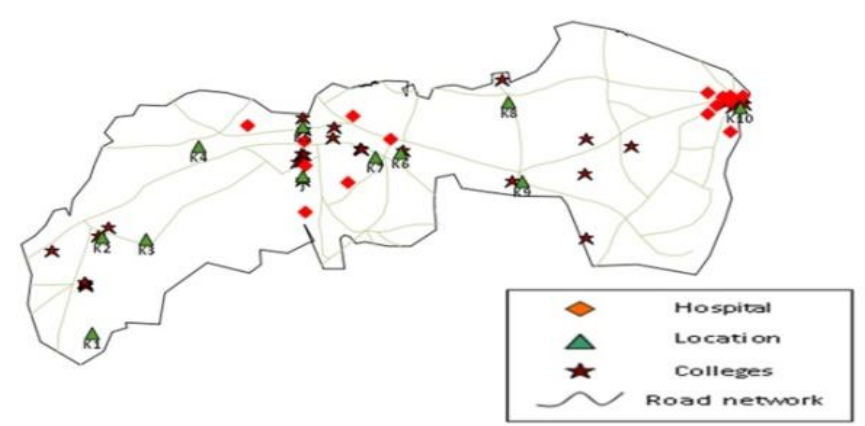

Figure. 3 Geographic space with $j$ and $k_{i}$ locations

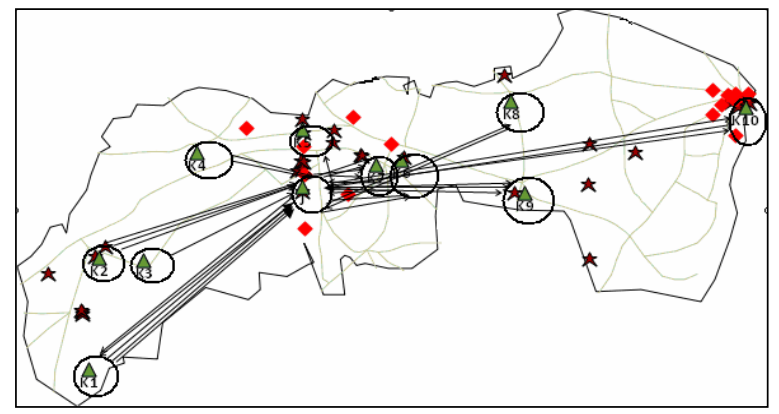

(a)

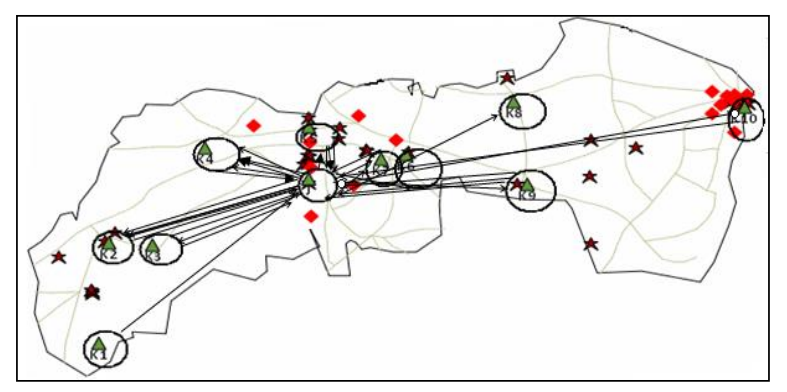

(c)

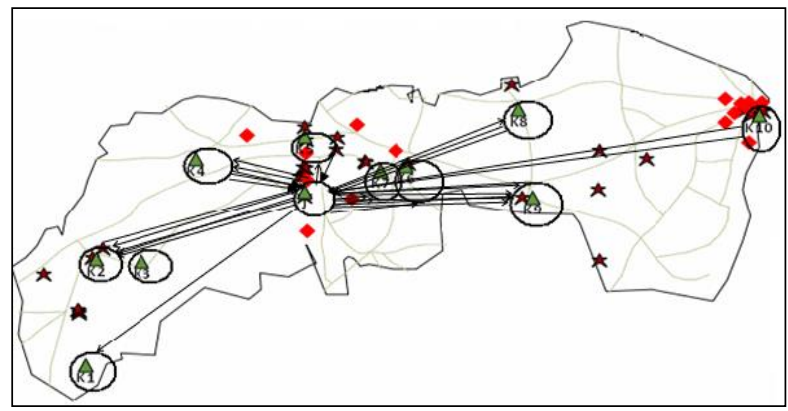

(b)

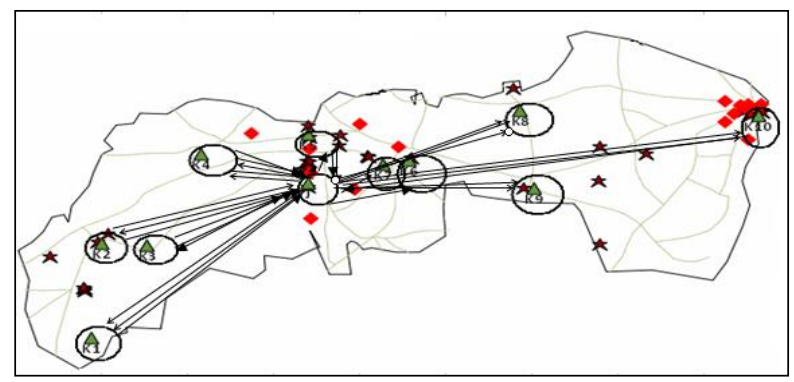

(d)

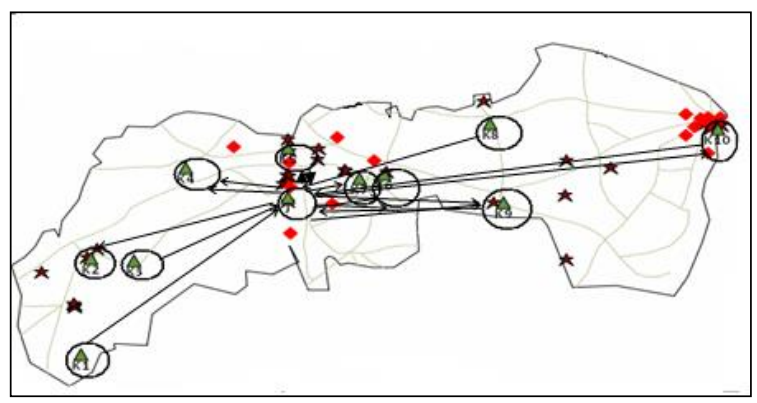

(e)

Figure 4 represents the mobility of individual at different time slice. Figure. 4(a) denotes the mobility of the infective individual at time period $\left(\mathrm{t}_{1}\right)$, Figure. 4(b) - Mobility of the infective individual at time period ( $\left.\mathrm{t}_{2}\right)$, Figure. 4(c) - Mobility of the infective individual at time period ( $\left.\mathrm{t}_{3}\right)$, Figure. $4(\mathrm{~d})$ - Mobility of the infective individual at time period ( $\left.\mathrm{t}_{4}\right)$, Figure. $4(\mathrm{e})$ - Mobility of the infective individual at time period $\left(\mathrm{t}_{5}\right)$ 
Table 4. Probability distribution for mobility of infected individual

\begin{tabular}{|l|l|l|}
\hline $\begin{array}{l}\text { Time } \\
\text { period }\end{array}$ & $\begin{array}{l}\text { Probability } \\
\text { value }\end{array}$ & Location \\
\hline$t_{1}$ & 0.12 & $j \rightarrow k_{3}$ \\
\hline$t_{2}$ & 0.75 & $j \rightarrow k_{9}$ \\
\hline$t_{3}$ & 0.52 & $k_{3} \rightarrow j$ \\
\hline$t_{4}$ & 0.32 & $k_{4} \rightarrow j$ \\
\hline$t_{5}$ & 0.10 & $j \rightarrow k_{9}$ \\
\hline
\end{tabular}

The numbers of people moving from source to destination location at various time slices are recorded. When the initial infected population in each location is defined, the number of individuals in an infected compartment $\left(\Delta C_{j}^{[i n f]}\right.$ ) for each subpopulation is calculated using

$\Delta C_{j}^{[m]}=\sum_{n}\left\{-D_{j}\left([m],[n]+D_{j}[n][m]\right)\right\}$

A multinomial distribution analysis is performed using MATLAB. For each time slice the probability of individuals moving from the source to each destination location in the infected compartment is determined. Finally the maximum probability value at each time slice is taken into account. Table 4 shows the routes with high probability distribution value. The probability distribution for mobility of infected individuals seems to be high for the time slices $t_{2}$ to $t_{4}$ in locations $k_{3}, k_{4}$ and $k_{9}$.

The spatio-temporal mobility pattern is derived using multinomial distribution model where the probability of people moving from one location to another location is generated with respect to dynamics of disease. As a result, the location that has a frequent mobility of individual in each compartment for various time slices is calculated. In order to verify the validity of the model it is compared with the result of mathematical model. The model based on differential equations defines the rate of individual present at that particular time but it fails to analyze the rate of change based on location. The mobility pattern on location is more effective when compared on pattern derived from human due to its complexity to design the human patterns. The locations that have frequent mobility are ranked and is more useful for the district administrators to make decision on prevention strategies. The time slice $\left(\mathrm{t}_{2}\right)$ is the peak time period where there are more number of people moving from $\mathrm{j}$ location to $\mathrm{k}_{\mathrm{i}}$ locations in infected compartment.

To visualize and simulate the mobility distribution of the input dataset, we use Quantum GIS (1.7.1) software. It is used to overlay road network map, source and destination location map, college map, hospital map and mobility of infected population over geographical space map (considered for the study) (Figure 3). The resultant map is then analyzed to determine the mobility of the infected individuals at different time slices (Figure 4(a) Figure 4(e)). The results clearly depicts that there is a high mobility during the time periods $t_{2}$ to $t_{4}$ (Figure 4(b), Figure 4(c) and Figure 4(d)) which is similar to values derived from multinomial distribution. The experiment shows that the mobility distribution pattern identified using multinomial distribution is effective.

However, this analysis shows that differences in commuting networks could lead to changes in spatial spread. For example, the random distribution is not considered for the analysis. If the infected individual starts form the source location, but did not reach the destination location. Instead of using smoothed version based on a gravity model, we used the raw commuting data from the census. As our data collected was exhaustive, preprocessing of data was not necessary. Even though using raw data leads to more heterogeneity in commuting links, it may also lead to results that are very dependent on the reported mobility that captures only a part of human mobility. Most of the mobility pattern studies have used SIR and SEIR compartmental models where these models fails to analyse the treatment compartment and also the mobility rate is not included in these models. In the proposed SLITR model, various compartments are designed and distribution of mobility pattern can be derived for any compartment.

In order to find the effectiveness of the proposed SLITR model, transmission rate is calculated for all the epidemic models such as SIR, SEIR based on two approaches namely differential and distributive approach. The transmission rate (TR) is the percentage of the possible contacts that results in the disease being spread. It calculates the number of people who get infected per time period by the infectious person. The outbreak of disease is identified by reproduction number $\left(\mathrm{R}_{0}\right)$. If the reproduction number is less than 1 , then the spread of infection will die out where the removal rate is greater than the infection rate. If the reproduction number is greater than one, then the infection will be able to spread in population where the recovery rate is greater than the infection rate. To control the 
spread of disease the infection or transmission rate must be minimized. The value generated using transport operator from Equation 23 is used as mobility rate or contact rate for SLITR model. The transmission rate $(\mathrm{TR}=0.29)$ calculated in time slice $t_{2}$ (Table 5) is greater than other time slice. In the same time slice the $\mathrm{TR}=0.16$ and $\mathrm{TR}=0.20$ for the models SIR and SEIR. As a result the proposed model is closer to the real dataset $(\mathrm{TR}=0.33)$ that is considered for this study and also proves the significance of mobility factor for the modelling of disease. This inference also shows that the mobility is directly proportional to the spread of disease.

These insights and findings not only captures human mobility relevant for the geographical epidemic spread, but they may pave the way for modelling important dynamical process in human and animal epidemiology. Thus the commuting data that is used to describe the population mobility in epidemic models is highly correlated with the spread of epidemic disease.

Table 5. Transmission rate of different epidemic models

\begin{tabular}{|l|l|l|l|l|}
\hline \multirow{2}{*}{$\begin{array}{l}\text { Time } \\
\text { slice }\end{array}$} & \multicolumn{4}{|c|}{ Transmission Rate (TR) } \\
\cline { 2 - 5 } & SIR & SEIR & SLITR & $\begin{array}{l}\text { Observed } \\
\text { Dataset }\end{array}$ \\
\hline $\mathbf{t}_{\mathbf{1}}$ & 0.025 & 0.042 & 0.067 & 0.08 \\
\hline $\mathbf{t}_{\mathbf{2}}$ & 0.16 & 0.20 & 0.29 & 0.33 \\
\hline $\mathbf{t}_{\mathbf{3}}$ & 0.108 & 0.148 & 0.150 & 0.192 \\
\hline $\mathbf{t}_{\mathbf{4}}$ & 0.06 & 0.075 & 0.108 & 0.125 \\
\hline $\mathbf{t}_{\mathbf{5}}$ & 0.01 & 0.033 & 0.05 & 0.067 \\
\hline
\end{tabular}

\section{Conclusion}

The paper focuses on identifying the mobility of an infected individual to predict the transmission rate of H1N1 a pandemic that occurred first in 2009. Initially a compartmental model is developed that categorizes the individuals who travel and who do not travel. Further to this, dynamics of disease is predicted using a distributive approach. Travel pattern of the infected individual plays a key role in predicting the spread of disease over space and time. The transmission of the proposed model is compared with other epidemic models and is observed that SLITR transmission rate is very much closer to the actual transmission rate, which is $87 \%$ accurate whereas SIR and SEIR is $48 \%$ and $60 \%$ respectively over all time slices. This analysis will be very much useful for policy decision makers on designing prevention and control strategies. Our future work considers the social structure and characteristics of the infected individuals for designing decision support system.

\section{References}

[1] L. Hufnagel, D. Brockmann and T. Geisel, "Forecast and control of epidemics in a globalized world", Proceedings of the National Academy of Sciences of the United States of America, Vol. 101, No.42, pp. 15124-15129, 2004.

[2] A. Flahault, E. Vergu, L. Coudeville and R. Grais, "Strategies to conducting a global influenza pandemic", Vaccine, Vol. 24, pp. 6751-6755, 2006.

[3] World Health Organization, Influenza: Fact sheet, http://www.who.int/csr/disease/swineflu/en

[4] B. D. Dalziel, B. Pourbohloul and S. P. Ellner, "Human mobility patterns predict divergent epidemic dynamics among cities", Proceedings of the Royal Society of London B: Biological Sciences, Vol. 280, 20130763, 2013.

[5] H. Malchow, S. Petrovskii, and E. Venturino, Spatio temporal patterns in ecology and epidemiology: theory, models, and simulation. London: Chapman \& Hall/CRC, 2008.

[6] H. Yang, S. Parthasarathy and S. Mehta, "A generalized framework for mining spatio-temporal patterns in scientific data", In Proceedings of the eleventh ACM SIGKDD International Conference on Knowledge discovery in data mining, pp. 716721, 2008.

[7] Y. Huang, L. Zhang and P. Zhang, "A framework for mining sequential patterns from spatio-temporal event data sets", IEEE Transactions on Knowledge and Data Engineering, Vol. 20, No. 4, pp. 433-448, 2008.

[8] W.O. Kermack, "A contribution of the Mathematical Theory of Epidemics", Proceedings of the Royal Society, Vol. 115, pp. 700-72, 1927.

[9] S. A. Karim and R. Razali, "A proposed Mathematical Model of Influenza A, H1N1 for Malaysia", Journal Applied Sciences, Vol. 11, pp. 1457-1460, 2011.

[10] L. Rvachev and I. M. Longini, "A mathematical model for the global spread of influenza", Mathematical biosciences, Vol. 75, No. 1, pp.3-22, 1985.

[11] H. C. Tuckwell and R. J. Williams, "Some properties of a simple stochastic epidemic model of SIR type", Mathematical biosciences, Vol. 208, No.1, pp.76-97, 2007.

[12] E. Frias-Martinez, G. Williamson and V. FriasMartinez, "An agent-based model of epidemic spread using human mobility and social network information", IEEE Third International Conference on Social Computing (SocialCom),pp. 57-64, 2011

[13] L. Perez, and S. Dragicevic, "An agent-based approach for modeling dynamics of contagious disease spread", International journal of health geographics, Vol. 8, No. 1, 2009. 
[14] K.M. Khalil, M. Abdel-Aziz, T.T. Nazmy and A. Salem, "An Agent-Based Modeling for Pandemic Influenza in Egypt", CoRR abs/1001.5275, 2010.

[15] Y. Zhang, Z. Liu, Y. Zhang, H. Yang, L. Fang and X. Xiao, "Spatially explicit epidemiological simulation system of influenza A (H1N1) in China", 18th International Conference on Geoinformatics, pp. 1-6, 2010.

[16] Yang, Kang-Hung and Jin-Yuan Hsu, "A new SIRbased model for influenza epidemic", World Academy of Science, Engineering and Technology, Vol. 6, 2012.

[17] C. Sun, Y.H. Hsieh, "Global analysis of an SEIR model with varying population size and vaccination", Applied Mathematical Modelling, Vol. 34, No. 10, pp. 2685-2697, 2010.

[18] M. Marder, "Dynamics of epidemics on random networks", Physical Review E, Vol. 75, pp. 066103/1-5, 2007.

[19] D. Balcan, B. Gonçalves, H. Hu, J. J. Ramasco, V. Colizza, and A. Vespignani, "Modeling the spatial spread of infectious diseases: The GLobal Epidemic and Mobility computational model", Journal of computational science, Vol. 1, No. 3, pp. 132-145, 2010.

[20] S. Merler and M. Ajelli, "Human mobility and population heterogeneity in the spread of an epidemic", Procedia Computer Science, Vol. 1, No. 1, pp. 2237-2244, 2010.

[21] L. Sattenspiel and K. Dietz, "A structured epidemic model incorporating geographic mobility among regions", Mathematical biosciences, Vol. 128, No. 1, pp. 71-91, 1995.

[22] V. Belik, T. Geisel and D. Brockmann, "Natural human mobility patterns and spatial spread of infectious diseases", Physical Review X, Vol. 1, No. 1, 011001, 2011

[23] S. Eubank, H. Guclu., V.S. Anil Kumar, M.V. Marathe, A. Srinivasan, Z. Toroczkai, N. Wang, "Modelling disease outbreaks in realistic urban social networks", Nature, Vol. 429, pp. 180-184, 2004.

[24] B. Wang, L. Cao, H. Suzuki and K. Aihara, "Safety-information-driven human mobility patterns with metapopulation epidemic dynamics", scientific reports, 2, 2012.

[25] D. Balcan, V. Colizza, B. Gonçalves, H. Hu, J. Ramasco and A. Vespignani, "Multiscale mobility networks and the spatial spreading of infectious diseases", Proceedings of the National Academy of Sciences, Vol. 106, No.51, pp. 21484-21489, 2009.

[26] S. Riley, "Large-scale spatial-transmission models of infectious disease", Science, Vol. 316, pp. 12981301, 2007.

[27] S. Charaudeau, K. Pakdaman and P. Y. Boelle, "Commuter mobility and the spread of infectious diseases: application to influenza in France", PloS one, Vol. 9, No. 1, e83002, 2014.
[28] L. Goldman and A. I. Schafer, Cecil Medicine: Expert Consult-Online. Elsevier Health Sciences, 2011.

[29] A. M. Mavinkurve-Groothuis, M. van der Flier, F. Stelma, C. van Leer-Buter, F. W. Preijers and P. M. Hoogerbrugge, "Absolute lymphocyte count predicts the response to new influenza virus $\mathrm{H} 1 \mathrm{~N} 1$ vaccination in pediatric cancer patients", Clinical and Vaccine Immunology, Vol. 20, No. 1, pp. 118121, 2013.

[30] H. Kesten, and N. Morse, "A property of the multinomial distribution", The Annals of Mathematical Statistics, Vol. 30, No. 1, pp. 120-127, 1959. 